\title{
A influência da cobertura vegetal e da distância do remanescente florestal no processo de regeneração natural na Floresta Ombrófila Densa Montana ${ }^{1}$
}

\author{
$\underline{\text { Silvana Cristina Pereira Muniz de Souza }}{ }^{2,4}$, Sergius Gandolfi ${ }^{3}$ e Ricardo Ribeiro Rodrigues ${ }^{3}$
}

Recebido: 13.04.2017; aceito: 19.12.2017

\begin{abstract}
The influence of the vegetation cover and the remnant forest distance in the natural regeneration process in a Dense Montane Ombrophilous Forest). Considering the large number of degraded and abandoned areas resulting from plantation and pasture activities, this study aimed to evaluate the effect of land use and distance of a forest remnant on natural regeneration in the Jurupará State Park, where pasture and citrus plantation were abandoned nine years ago. It was evaluated the density of seed rain, seed bank and saplings in these past land uses. Three transects of $300 \times 20 \mathrm{~m}$ were placed, with 15 delimited plots of $20 \times 20 \mathrm{~m}$; from each transect five of these plots were surveyed. The past land use significantly influenced the seed rain, which presented higher density in the ex-citrus plantation in relation to the pasture. The latter, however, presented no significant effect on the variables analyzed. The ex-citrus plantation serves as a catalyst for the natural regeneration process, while the pasture requires management to reduce grass competition with natural regeneration. Keywords: resilience, saplings, seed bank, seed rain
\end{abstract}

RESUMO - (A influência da cobertura vegetal e da distância do remanescente florestal no processo de regeneração natural na Floresta Ombrófila Densa Montana). Considerando o grande número de áreas degradadas e abandonadas provenientes de atividades agropastoris, este estudo teve como objetivo avaliar o efeito do uso do solo com pastagem e citricultura interrompido há nove anos, e da distância do remanescente florestal na regeneração natural no Parque Estadual do Jurupará. Avaliamos a densidade da chuva de sementes, banco de sementes e indivíduos jovens. Foram instalados três transectos de $300 \times 20 \mathrm{~m}$ e delimitadas 15 parcelas de $20 \times 20 \mathrm{~m}$, sendo sorteadas cinco parcelas de cada transecto. $\mathrm{O}$ tipo de cobertura vegetal foi significativo para chuva de sementes, que apresentou maior densidade na área de citricultura em relação à pastagem. Na pastagem, a distância do remanescente florestal não foi significativa para os parâmetros analisados. A área de citricultura atuou como catalisadora do processo de regeneração natural, enquanto a pastagem necessita de manejo para reduzir a competição das gramíneas com a regeneração natural.

Palavras-chave: banco de sementes, chuva de sementes, indivíduos jovens, resiliência

\section{Introdução}

No Brasil a exploração desordenada de seus recursos naturais resultou em milhões de hectares de áreas desflorestadas convertidas em pastagens, lavouras e centros urbanos (Rodrigues et al. 2009). Em especial, as áreas destinadas as atividades agropastoris são frequentemente abandonadas devido ao declínio na produtividade e mudança nos incentivos econômicos (Uhl et al. 1988, Gandolfi et al. 2007a) e, por sua vez, têm geralmente baixa resiliência, particularmente aquelas que sofreram intensa perturbação com a utilização de agricultura mecanizada (Liebsch et al. 2008).

Diante desse panorama, estudos que avaliam o potencial de regeneração natural tanto de pastagens, como de plantios comerciais são de extrema importância para aumentar a efetividade da recuperação e diminuir os custos da recuperação destas áreas, pois a identificação da metodologia de restauração

1. Parte da dissertação de Mestrado do primeiro Autor

2. Instituto Florestal, Rua do Horto, 931, 02377-000 São Paulo, SP, Brasil

3. Universidade de São Paulo, Departamento de Ciências Biológicas, Laboratório de Ecologia e Restauração Florestal, Avenida Pádua Dias, 11, São Dimas, 13418260 Piracicaba, SP, Brasil

4. Autor para correspondência: silvana1souza@gmail.com 
mais adequada para uma dada área depende de um diagnóstico apropriado do local a ser restaurado e do entorno imediato e regional (Rodrigues et al. 2009).

Viani et al. (2010), em uma compilação dos principais resultados de estudos que tratam da regeneração natural sob plantios florestais, observou a tendência de que as plantações florestais comerciais podem ser catalisadores da regeneração natural das espécies arbustivas e arbóreas nativas, sendo estas áreas, em determinadas circunstâncias, redutos de biodiversidade. $\mathrm{O}$ que se verifica na reconstrução das comunidades nativas sob as plantações florestais é que, assim como em florestas nativas, há diversos filtros cuja importância relativa se altera no espaço e no tempo e que determinam as regras de montagem, "assembly rules", da comunidade em regeneração e, portanto, o caminho que será seguido na sucessão (Bossuyt et al. 2005).

Já em pastagens abandonadas, têm sido observados vários fatores que limitam o estabelecimento de espécies florestais, Aide (2000) ao investigar as barreiras para a regeneração natural de pastagens abandonadas em Porto Rico verificou que os maiores fatores eram a perda da chuva de sementes e, consequentemente, do banco de sementes. Os estudos de Ingle (2003) e Dosh et al. (2007) evidenciaram que a chuva de sementes apresenta um padrão espacial agregado, indicando que a dispersão tende a declinar logaritmicamente com o aumento da distância das matrizes e, Slocum \& Horvitz (2000) observaram que os agentes dispersores, tanto mamíferos, quanto aves; evitam áreas limpas devido ao risco de predação; desta forma, a chuva de sementes de espécies zoocóricas pode estar negativamente correlacionada com a distância da borda da floresta. Já para as espécies anemocóricas, Greene \& Johnson (1996) sugerem que o número de sementes dispersas pelo vento declina em relação à distância da borda da floresta. Segundo Holl (1999) quando não é observada tal tendência, as sementes encontradas nos coletores são geralmente de espécies já presentes nas suas imediações ou nos seus arredores.

Ainda, considerando as plantações comerciais, Viani et al. (2010) sugere que há uma lacuna de conhecimento na quantificação de indicadores de regeneração natural sob estes plantios. Nos últimos anos, poucos estudos foram publicados com o objetivo de quantificar a regeneração natural, tanto em plantios comerciais, como em pastagens abandonadas.

$\mathrm{O}$ fato de que árvores e arbustos, independente da sua origem (nativas ou exóticas) promovem uma efetiva rede facilitadora da dispersão e dos estágios iniciais de estabelecimento de indivíduos jovens; e de que o aumento da distância da borda do remanescente florestal diminui a dispersão e consequentemente o estabelecimento de jovens, testamos neste estudo as seguintes hipóteses: i) a densidade da chuva de sementes, banco de sementes e indivíduos jovens é maior em áreas com cobertura florestal (citricultura) do que em áreas com gramíneas exóticas (pastagem); ii) em áreas de pastagem, a densidade da chuva, banco de sementes e indivíduos jovens diminui com aumento da distância da borda do remanescente florestal.

\section{Material e métodos}

Área de estudo - O Parque Estadual do Jurupará (PEJU) está localizado na região do Vale do Ribeira, Estado de São Paulo, sobre o Planalto Atlântico (Lima \& Aguirre 2010). Abrange os municípios de Ibiúna e Piedade, com uma área de 26.250 ha entre as coordenadas geográficas $23^{\circ} 51^{\prime}$ 'a $24^{\circ} 02^{\prime}$ 'S e $47^{\circ} 11^{\prime}$ 'a $47^{\circ} 24^{\prime} \mathrm{W}$ (figura 1). O clima na região é classificado como mesotérmico brando, quente de inverno seco, Cfb segundo a classificação de Köppen (1948), com temperaturas médias no inverno entre 15 e $17^{\circ} \mathrm{C}$, enquanto que no verão oscilam em torno dos $22^{\circ} \mathrm{C}$ (Fundação Florestal 2010). A precipitação anual média dos últimos 43 anos foi de $1713 \pm 350 \mathrm{~mm}$ (Posto

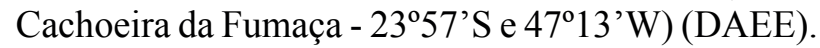
Predominam na área rotas de ventos de sudeste em decorrência da predominância da circulação da massa Tropical Atlântica e sistemas frontais (Fundação Florestal 2010).

A área experimental localiza-se na borda de um remanescente florestal de Floresta Ombrófila Densa Montana. As alterações antrópicas desta área foram registradas a partir de 1970 e estas se intensificaram a partir de 1985, com o corte da floresta nativa,

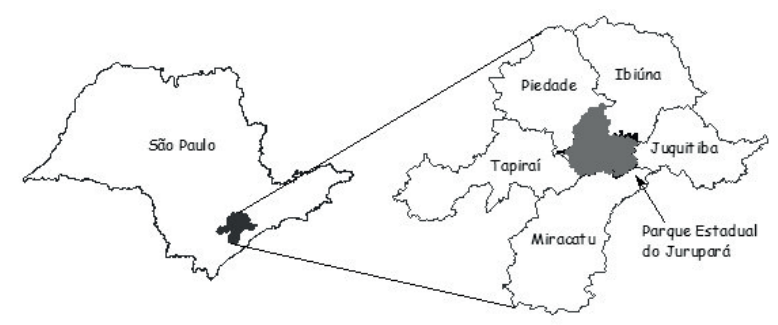

Figura 1. Mapa esquemático com a localização do Parque Estadual do Jurupará, município de Ibiúna, São Paulo.

Figure 1. Schematic map of the Jurupará State Park, municipality of Ibiúna, São Paulo State. 
seguido de queima. Logo após o desmatamento, estas áreas foram destinadas a atividades agropastoris, em especial citricultura e pastagem (Lima \& Aguirre 2010). No momento da instalação desse experimento, a área estava com nove anos de abandono.

A área de citricultura ocupa uma estreita faixa (40 m) entre o remanescente florestal e uma estrada desativada, totalizando aproximadamente dois hectares. Consiste no cultivo em linha de Citrus latifolia Tanaka (limão-taiti) em enxertia sob a espécie Citrus limonia L. Osbeck (limão-cravo) e apresenta estágio inicial de regeneração natural, com um dossel de aproximadamente $3 \mathrm{~m}$ de altura.

A área de pastagem com 10 hectares, embora abandonada na mesma época que a área de citricultura, não foram observados sinais de regeneração natural condizente com o tempo de abandono, apresentando uma cobertura vegetal constituída predominantemente pelas espécies inicialmente introduzidas.

Coleta de dados - A área experimental total consistiu de três transectos de $20 \times 300 \mathrm{~m}$, distribuídos da seguinte forma: um transecto no interior da área de citricultura (C); dois outros transectos na pastagem, sendo um à distância de 0 a $20 \mathrm{~m}$ da borda do remanescente florestal (P1) e o outro à distância de 80 a $100 \mathrm{~m}$ da borda do remanescente florestal (P2). Em cada transecto foram delimitadas 15 parcelas de $20 \times 20 \mathrm{~m}$ e, destas, sorteadas cinco parcelas amostrais (figura 2).

Para a avaliação da chuva de sementes foram distribuídos aleatoriamente em cada parcela três coletores de sementes, totalizando 15 coletores em cada transecto. Foram utilizados coletores circulares com abertura de $0,52 \mathrm{~m}$ diâmetro, totalizando uma área de $0,21 \mathrm{~m}^{2}$ por coletor. Com o intuito de coletar sementes de todos os tamanhos e evitar acúmulo de umidade, foi fixado na abertura dos coletores um tecido com a composição de $70 \%$ de poliéster e $30 \%$ de algodão (Grombone-Guaratini 1999). Mensalmente, durante o período de um ano, os coletores tiveram o seu conteúdo recolhido. O material coletado foi triado separando-se manualmente as sementes do restante da serapilheira.

Para avaliação do banco de sementes foram coletadas três amostras ao acaso de cada parcela sorteada, totalizando 15 amostras em cada transecto. Cada unidade amostral do banco de sementes consistia de uma camada de solo de $0,25 \times 0,25 \mathrm{~m}\left(0,0625 \mathrm{~m}^{2}\right)$, com $0,05 \mathrm{~m}$ de profundidade. Foram realizadas duas coletas do banco de sementes, em janeiro e agosto do mesmo ano. Utilizamos o método de incubação do solo, para quantificar o número de sementes viáveis, considerando a emergência de plântulas (Heerdt et al. 1996). O solo coletado foi acomodado em bandejas no viveiro do PEJU, sob condições de alta luminosidade (70\% da luminosidade total) e três irrigações diárias. $\mathrm{O}$ registro da germinação de plântulas foi realizado mensalmente, pelo período de seis meses para cada coleta. Para confirmar a identificação botânica das plantas germinadas, pelo menos um indivíduo de cada espécie registrada foi transplantado para sacos de polietileno e o seu desenvolvimento acompanhado até que fosse possível herborizar o material para uma identificação segura.

O estrato regenerante foi avaliado a partir de 4 subparcelas aleatórias permanentes de $0,5 \times 0,5 \mathrm{~m}$ por parcela, totalizando 20 subparcelas e $5 \mathrm{~m}^{2}$ em cada transecto. $\mathrm{O}$ critério de amostragem incluiu indivíduos jovens cujas alturas estiveram entre 0,30 e $1,30 \mathrm{~m}$. Foram realizadas três avaliações: fevereiro, agosto e fevereiro, completando um ano de acompanhamento.

A identificação e a classificação de todo o material foi realizada mediante consulta a especialistas, material de herbário e literatura especializada. A classificação das famílias foi baseada em APG IV (Angiosperm Phylogeny Group 2016). Após a identificação do material, as espécies foram classificadas por forma de vida baseado na divisão de Aubréville (1963), mas adaptada de acordo com as observações de campo, definidas a seguir: i) árvore - planta lenhosa, com altura igual ou superior a $3 \mathrm{~m}$, ii) arbusto - planta pequena e de base lenhosa, que ramifica abaixo de

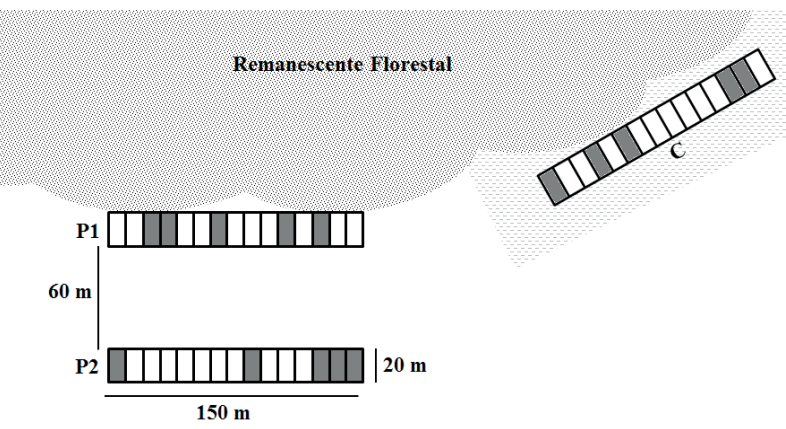

Figura 2. Esquema representando a distribuição dos transectos e das parcelas na paisagem no Parque Estadual do Jurupará. Em destaque (cinza) as parcelas sorteadas. C: área de citricultura, P1: transecto na pastagem de $0-20 \mathrm{~m}$ de distância do remanescente florestal, P2: transecto na pastagem de 80-100 m de distância do remanescente florestal.

Figure 2. Schematic representation of transects and plots within the landscape of the Jurupará State Park. In gray, randomized plots. C: ex-citrus plantation, P1: transect in ex-pasture 0-20 m from the forest remnant, $\mathrm{P} 2$ : transect in ex-pasture 80-20 $\mathrm{m}$ from the forest remnant. 
0,3 m de altura, iii) erva - toda a planta herbácea e terrestre, que compõe o estrato herbáceo, iv) liana para esta categoria foi utilizada a definição de Kim (1996), para a qual o termo liana refere-se a toda planta de hábito escandente de forma ampla, tanto herbácea quanto lenhosa. Todas as espécies também foram classificadas pela síndrome de dispersão das sementes - espécies zoocóricas, anemocóricas e autocóricas - com base em van der Pijl (1982). As espécies foram ainda classificadas quanto à origem em nativas e exóticas. As espécies consideradas exóticas e "naturalizadas" pela Lista de Espécies da Flora do Brasil (2016) foram excluídas dos cálculos estatísticos e histogramas.

Análises de dados - Para os cálculos estatísticos foram considerados o número de indivíduos de espécies nativas em cada amostra da chuva de sementes, banco de sementes e estrato regenerante, observando-se a existência de diferenças significativas entre as seguintes situações: $\mathrm{C} \times \mathrm{P} 1$ e $\mathrm{P} 1 \times \mathrm{P} 2$. O número de indivíduos não apresentaram normalidade, nem homogeneidade das variâncias, verificados respectivamente, a partir dos testes de Shapiro-Wilk e de análise de homogeneidade das variâncias. Os dados transformados em raiz quadrada também não apresentaram normalidade, nem homogeneidade das variâncias. Considerando que os pressupostos de normalidade e homogeneidade das variâncias não foram atendidos, aplicamos aos dados originais o teste não paramétrico de Mann-Whitney, assumindo $\alpha=0,05$, como sugerido em Dalgaard (2002). Todas as análises foram feitas usando o pacote estatístico "R" (R Core Team 2016).

\section{Resultados}

A lista das espécies amostradas e a densidade absoluta correspondente a chuva de sementes, banco de sementes e estrato regenerante são apresentadas na tabela 1 para a área $\mathrm{C}$ e na tabela 2 para as áreas $\mathrm{P} 1 \mathrm{e}$ $\mathrm{P} 2$. Os dados quantitativos obtidos de cada parâmetro analisado nas três áreas foram sumarizados na tabela 3 .

Na avaliação da chuva de sementes, foi observado na área $\mathrm{C}$ um predomínio de espécies arbustivoarbóreas e da síndrome de dispersão zoocórica, já nas áreas P1 e P2 prevaleceram espécies herbáceas e a síndrome de dispersão anemocórica (figuras 3 e 4). Considerando as espécies nativas, a área $\mathrm{C}$ apresentou um maior número de sementes que a área $\mathrm{P} 1$,
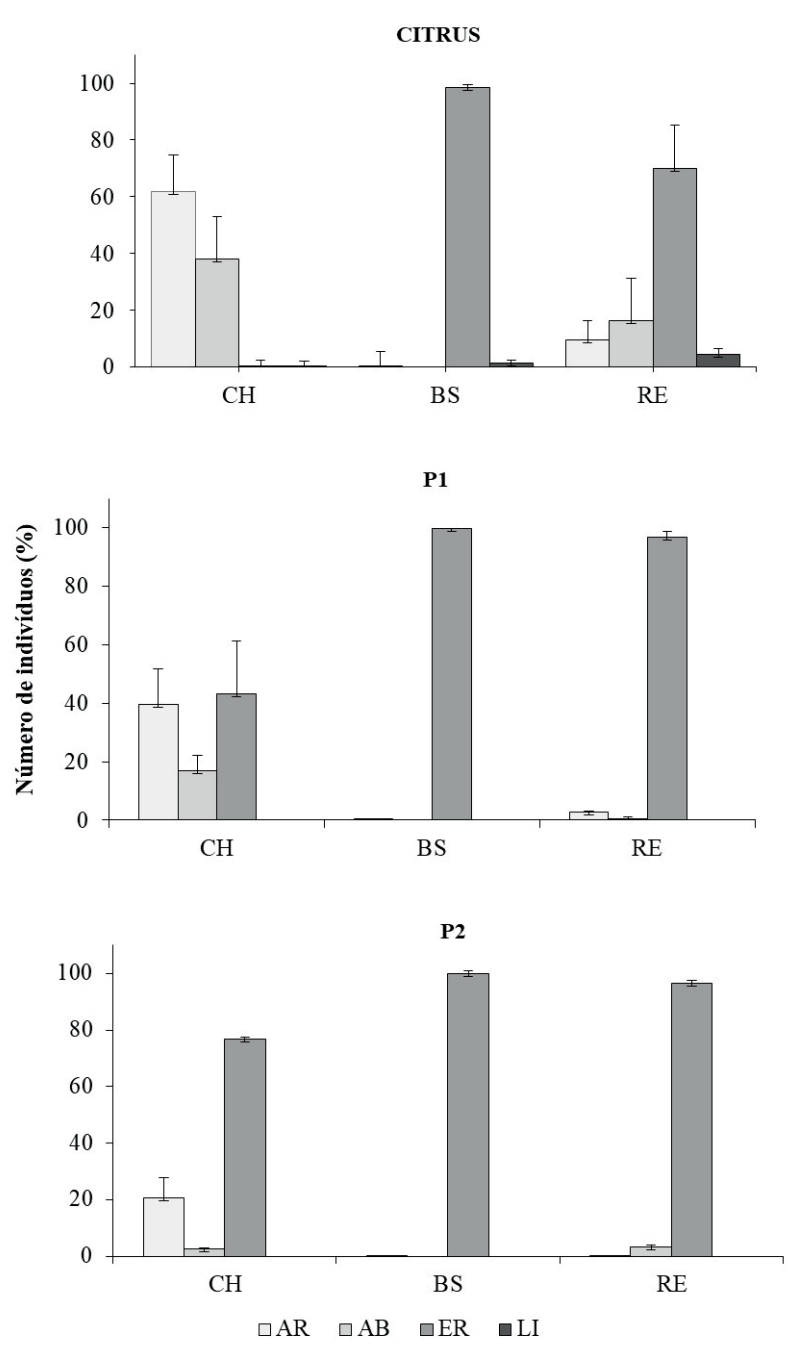

Figura 3. Porcentagem de formas de vida por parâmetro avaliado em cada área no Parque Estadual do Jurupará. AR: árvore, AB: arbusto, BS: banco de sementes, $\mathrm{CH}$ : chuva de semente, ER: erva, LI: liana, RE: estrato regenerante.

Figure 3. Percentage of life forms per parameter evaluated in each area of the Jurupará State Park. AR: tree, AB: shrub, BS: seed bank, $\mathrm{CH}$ : seed rain, ER: herb, LI: liana, RE: saplings.

resultando em diferenças significativas na avaliação da chuva de sementes entre as coberturas vegetais, $C$ e P1 $\left(U=225, p=1,28 \times 10^{-8}\right)$. Já entre as diferentes distâncias do remanescente na pastagem, P1 e P2, não foram encontradas diferenças significativas $(U=68$, $\mathrm{p}=0,07)$.

No banco de sementes, nas três áreas, foi observado um predomínio de espécies herbáceas e da síndrome de dispersão anemocórica (figuras 3 e 4). Considerando o número de sementes germinadas não foram encontradas diferenças significativas entre as coberturas vegetais, $\mathrm{C}$ e $\mathrm{P} 1,(\mathrm{U}=86, \mathrm{p}=0,28)$, nem entre as diferentes distâncias do remanescente na pastagem, $\mathrm{P} 1$ e $\mathrm{P} 2,(\mathrm{U}=108, \mathrm{p}=0,87)$. 

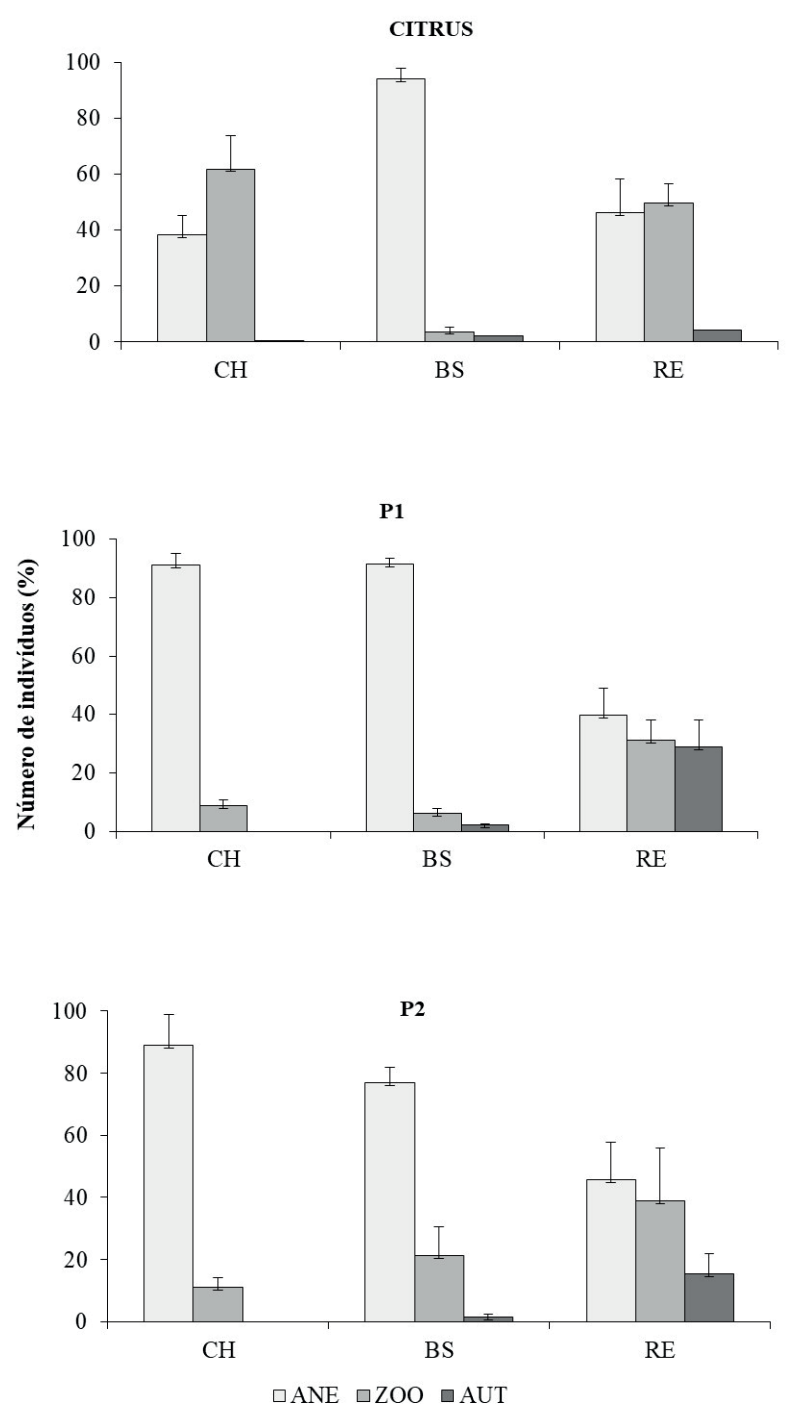

Figura 4. Porcentagem de síndrome de dispersão por parâmetro avaliado em cada área no Parque Estadual do Jurupará. ANE: anemocórica, AUT: autocórica, BS: banco de sementes, $\mathrm{CH}$ : chuva de sementes, RE: estrato regenerante, ZOO: zoocórica.

Figure 4. Percentage of dispersal syndrome per parameter evaluated in each area of the Jurupará State Park. ANE: anemochorous, AUT: autochorous, BS: seed bank $\mathrm{CH}$ : seed rain, RE: saplings, ZOO: zoochorous.

No levantamento do estrato regenerante nas três áreas foi observado um predomínio de espécies herbáceas, quanto a síndrome de dispersão, registramos o predomínio da zoocoria na área $\mathrm{C}$ e da anemocoria nas áreas P1 e P2 (figuras 3 e 4). A área P1 apresentou um maior número de indivíduos regenerantes que a área $\mathrm{C}$, em decorrência do predomínio das espécies herbáceas, resultando em diferenças significativas entre $\mathrm{C}$ e $\mathrm{P} 1(\mathrm{U}=97, \mathrm{p}=$ 0,005). Entre as diferentes distâncias do remanescente na pastagem P1 e P2 não foram encontradas diferenças significativas $(U=199, p=0,98)$.

\section{Discussão}

A cobertura vegetal foi determinante para a chuva de sementes, as espécies plantadas desempenharam, no sistema, papel semelhante ao de espécies pioneiras sob condições naturais (Tabarelli et al. 1993, SilvaJúnior et al. 1995, Holl et al. 2000). A presença de árvores plantadas em linhas como no presente estudo, em grupos, ou isoladas, promovem uma série de mudanças no ambiente, entre eles o provimento de habitat para dispersores de sementes, como observado por Camus et al. (2006) e Reid \& Holl (2013).

$\mathrm{Na}$ pastagem a distância do remanescente florestal, não influenciou quantitativamente a chuva de sementes. De uma forma geral, constatamos em ambas as distâncias, altas proporções de espécies com síndrome de dispersão anemocóricas, acima de $98 \%$, em contraposição ao proposto por Howe \& Smallwood (1982), que nas florestas tropicais a zoocoria é uma das síndromes mais frequentes com ocorrência mínima de $50 \%$, chegando até mais de $75 \%$. O predomínio em densidade e riqueza de espécies anemocóricas na pastagem pode estar relacionado à eventos estocásticos (Hubbell 2001); bem como, o possível direcionamento favorável do vento (Lopes et al. 2010). A ideia de que a presença de uma espécie em determinado local é definida, sobretudo, pela chegada de propágulos (teoria da "limitação da dispersão" - Obiri \& Lawes 2004) leva ao entendimento de que a falta de dispersores e o tamanho das sementes das espécies zoocóricas afetaria a capacidade destas espécies de alcançar naturalmente as pastagens degradadas, já que nesse caso, livre de obstáculos, as sementes pequenas e leves, geralmente anemocóricas, possuem maior eficiência na dispersão (Uhl et al. 1991, Lopes et al. 2010).

$\mathrm{O}$ distanciamento do remanescente florestal refletiu nas formas de vida, com o aumento da proporção de espécies herbáceas em detrimento das espécies arbóreas. A riqueza de espécies arbustivoarbóreas foi baixa tanto na pastagem (seis espécies), quanto na área de citricultura (nove espécies). Bertoncini \& Rodrigues (2008) também observaram baixa densidade e riqueza de espécies arbustivoarbóreas em uma pastagem abandonada, situada em uma matriz de Floresta Estacional Semidecidual. A dispersão primária e secundária envolve alta heterogeneidade espacial e temporal, diminuindo drasticamente a densidade de sementes dispersas a partir de $10 \mathrm{~m}$ de distância da borda do remanescente florestal em Holl (1999) e 2,5 m em Dosch et al. 
Tabela 1. Lista das espécies e valores de densidade absoluta encontrados na chuva de sementes, banco de sementes e estrato regenerante, no interior de uma área de citricultura abandonada no Parque Estadual do Jurupará, Ibiúna, SP. A: anemocórica, BS: banco de sementes, CS: chuva de sementes, DA: densidade absoluta (número de sementes $\mathrm{m}^{-2}$ ), RN: estrato regenerante (indivíduos $\mathrm{m}^{-2}$ ), SD: síndrome de dispersão, Z: zoocórica, U: autocórica. $\left(^{*}\right)$ espécie exótica.

Table 1. List of species and values of absolute density for seed rain, seed bank and saplings in an abandoned citrus plantation in Jurupará State Park, Ibiúna, SP. A: anemochorous, BS: seed bank, CS: seed rain, DA: absolute density (number of seed $\mathrm{m}^{-2}$ ), RN: saplings (individuals $\mathrm{m}^{-2}$ ), $\mathrm{SD}$ : propagule dispersal syndrome, $\mathrm{Z}$ : zoochorous, $\mathrm{U}$ : autochorous $\left(^{*}\right)$ introduced species.

\begin{tabular}{|c|c|c|c|c|}
\hline \multirow{2}{*}{ Espécies } & \multirow{2}{*}{ SD } & \multicolumn{3}{|c|}{ DA } \\
\hline & & $\mathrm{CS}$ & $\mathrm{BS}$ & $\mathrm{RN}$ \\
\hline \multicolumn{5}{|l|}{ Árvores } \\
\hline Alchornea triplinervia (Spreng.) Müll Arg. & $\mathrm{Z}$ & & 0,6 & 0,2 \\
\hline Cabralea canjerana (Vell.) Mart. & $\mathrm{Z}$ & & & 0,4 \\
\hline Citrus latifolia L.Osbeck & $\mathrm{Z}$ & & & 0,2 \\
\hline Clethra scabra Pers. & A & 3,1 & & 0,4 \\
\hline Ilex sp. & $\mathrm{Z}$ & 2,8 & & \\
\hline Miconia cabucu Hoehne & $\mathrm{Z}$ & & & 0,4 \\
\hline Myrcia multiflora (Lam.) DC. & $\mathrm{Z}$ & & 0,6 & 0,2 \\
\hline Ocotea odorifera (Vell.) Rohwer & $\mathrm{Z}$ & 0,9 & & \\
\hline Psidium guajava L.* & $\mathrm{Z}$ & 1,3 & & \\
\hline Myrsine coriacea (Sw.) R.Br. ex Roem. \& Schult. & $\mathrm{Z}$ & 24,5 & & 0,4 \\
\hline Senna multijuga (Rich.) H.S.Irwin \& Barneby & $\mathrm{U}$ & & 0,6 & \\
\hline Senna reticulata (Willd.) H.S.Irwin \& Barneby & $\mathrm{U}$ & & 0,6 & \\
\hline Tibouchina granulosa (Desr.) Cogn. & A & 11904,5 & & \\
\hline Trema micrantha (L.) Blume & $\mathrm{Z}$ & & 1,1 & \\
\hline Lessingianthus glabratus (Less.) H.Rob. & A & 18,2 & & \\
\hline Vernonanthura phosphorica (Vell.) H.Rob. & A & 747,1 & & \\
\hline Vitex megapotamica (Spreng.) Moldenke & $\mathrm{Z}$ & & & 0,2 \\
\hline \multicolumn{5}{|l|}{ Arbustos/subarbustos } \\
\hline Baccharis dracunculifolia DC. & A & 10,0 & & \\
\hline Leandra australis (Cham.) Cogn. & $\mathrm{Z}$ & 20658,2 & & 3,8 \\
\hline \multicolumn{5}{|l|}{ Ervas } \\
\hline Achyrocline satureioides (Lam.) DC. & $\mathrm{A}$ & 0,3 & & \\
\hline Ageratum conyzoides $\mathrm{L}$. & A & & 0,6 & \\
\hline Amaranthus spinosus L.* & $\mathrm{U}$ & & 4,3 & \\
\hline Andropogon bicornis L. & A & 2,5 & & \\
\hline Andropogon leucostachyus Kunth & A & 0,6 & & \\
\hline Asclepias curassavica $\mathrm{L}$. & A & & 0,6 & \\
\hline Baccharis crispa Spreng. & A & 3,8 & & \\
\hline Centella asiatica (L.) Urb. & $\mathrm{U}$ & & 8,6 & \\
\hline Chaptalia nutans (L.) Pol. & A & 0,6 & & \\
\hline Chloris gayana Kunth* & A & & 609,1 & \\
\hline Clidemia sp. & $\mathrm{Z}$ & & & 1,0 \\
\hline Coccocypselum $\mathrm{sp}$ & $\mathrm{Z}$ & & & 0,6 \\
\hline
\end{tabular}


Tabela 1 (continuação)

\begin{tabular}{|c|c|c|c|c|}
\hline \multirow{2}{*}{ Espécies } & \multirow{2}{*}{$\mathrm{SD}$} & \multicolumn{3}{|c|}{ DA } \\
\hline & & $\mathrm{CS}$ & BS & RN \\
\hline Commelina erecta $\mathrm{L}$. & $\mathrm{A}$ & 9,4 & 259,2 & 1,0 \\
\hline Commelina sp. & A & & & 9,6 \\
\hline Conyza bonariensis (L.) Cronquist & A & 3,5 & & \\
\hline Cuphea carthagenensis (Jacq.) J.Macbr. & A & & 22,4 & \\
\hline Desmodium adscendens (SW.) DC.* & $\mathrm{Z}$ & 1,3 & & \\
\hline Borreria palustris (Cham. \& Schultdl.) Bacigalupo \& E.L.Cabral & A & 0,9 & 159,0 & \\
\hline Elephantopus mollis Kunth & A & 0,3 & & \\
\hline Coniza bonariensis (L.) Cronquist & A & & 2,2 & \\
\hline Chromolaena laevigata (Lam.) R.M.King \& H.Rob. & A & 62,5 & 10,2 & \\
\hline Chromolaena squalida (DC.) R.M. King \& H.Rob. & A & 0,6 & & \\
\hline Fimbristylis autumnalis (L.) Roem. \& Schult. & $\mathrm{A}$ & & 0,6 & \\
\hline Fimbristylis dichotoma (L.) Vahl & A & & 452,8 & \\
\hline Pterocaulon virgatum (L.) DC. & A & 0,3 & 0,6 & \\
\hline Pombalia communis (A St. -Hil.) Paula-Souza & $\mathrm{Z}$ & 5,7 & & \\
\hline Jaegeria hirta (Lag.) Less. & A & 0,6 & 4,3 & \\
\hline Melilotus indicus (L.) All. & $\mathrm{Z}$ & 0,9 & & \\
\hline Melinis minutiflora P. Beauv.* & A & & 0,6 & \\
\hline Panicum sp. & A & & 5,4 & \\
\hline Paspalum conspersum Schrad. & A & 0,9 & & \\
\hline Phyllanthus tenellus Roxb. & A & & 11,2 & \\
\hline Piper sp. & $\mathrm{Z}$ & & & 3,0 \\
\hline Rubus brasiliensis Mart. & $\mathrm{Z}$ & & 0,6 & 0,4 \\
\hline Scleria gaertneri Raddi & A & & & 0,2 \\
\hline Scoparia dulcis L. & $\mathrm{A}$ & & 1,6 & \\
\hline Sida rhombifolia $\mathrm{L}$. & $\mathrm{Z}$ & & 33,1 & 1,0 \\
\hline Solanum americanum Mill. & $\mathrm{Z}$ & 21,0 & & \\
\hline Urochloa decumbens (Stapf) R.D.Webster* & A & 1,3 & 2,2 & \\
\hline Urochloa mutica (Forssk.) T.Q. Nguyen* & $\mathrm{A}$ & 0,3 & 67,2 & \\
\hline \multicolumn{5}{|l|}{ Lianas } \\
\hline Aristolochia $\mathrm{sp.}$ & $\mathrm{U}$ & & 12,3 & 1,0 \\
\hline Ipomoea sp. & $\mathrm{U}$ & 8,2 & & \\
\hline \multicolumn{5}{|l|}{ Indeterminadas } \\
\hline Baccharis sp. & $\mathrm{A}$ & 13,8 & & \\
\hline Vernonia sp. & A & 0,3 & & \\
\hline
\end{tabular}

(2007), prevalecendo espécies típicas de pastagem, predominantemente herbáceas.

O banco de sementes estudado, de uma forma geral, apresentou 98\% das espécies herbáceas e síndrome de dispersão anemocórica. Poucas espécies arbóreas foram encontradas, todas em baixa densidade, sendo cinco espécies na área de citricultura: Alchornea triplinervia, Myrcia multiflora, Senna reticulata, Senna multijuga e Trema micrantha e, apenas duas espécies, na pastagem: Senna multijuga e Trema micranta.

A situação de dominância das espécies herbáceas é comum nos bancos de sementes de comunidades 
Tabela 2. Lista das espécies e valores de densidade absoluta encontrados na chuva de sementes, banco de sementes e estrato regenerante, na pastagem abandonada no Parque Estadual do Jurupará, Ibiúna, SP. P1: 0-20 m de distância do remanescente florestal, P2: 80-100 m de distância do remanescente florestal. A: anemocórica, BS: banco de sementes, CS: chuva de sementes, DA: densidade absoluta (número de sementes $\mathrm{m}^{-2}$ ), RN: estrato regenerante (indivíduos $\mathrm{m}^{-2}$ ), SD: síndrome de dispersão, Z: zoocórica, U: autocórica. (*) espécie exótica.

Table 2. List of species and values of absolute density for seed rain, seed bank and saplings in an abandoned pasture in Jurupará State Park, Ibiúna, SP. P1: 0-20 m distance from the forest remnant, P2: 80-100 m distance from the forest remnant. A: anemochorous, BS: seed bank, CS: seed rain, DA: absolute density (number of seeds $\mathrm{m}^{-2}$ ), RN: saplings (individuals $\mathrm{m}^{-2}$ ), SD: dispersion syndrome, Z: zoochorous, $\mathrm{U}$ : autochorous $\left({ }^{*}\right)$ introduced species.

Espécies/Species

\begin{tabular}{ccccccc} 
& \multicolumn{3}{c}{ SD } & & & \multicolumn{3}{c}{ P2 } \\
\cline { 2 - 7 } & & DA & & & DA & \\
\cline { 2 - 7 } & CS & BS & RN & CS & BS & RN
\end{tabular}

Árvores

Alchornea triplinervia (Spreng.) Müll Arg.

Clethra scabra Pers.

Miconia cabucu Hoehne

Psidium guajava L.*

Myrsine coriacea (Sw.) R.Br. ex Roem. \& Schult.

Senna multijuga (Rich.) H.S. Irwin \& Barneby

Tibouchina granulosa (Desr.) Cogn.

Trema micrantha (L.) Blume

Lessingianthus glabratus (Less.) H.Rob.

Vernonanthura phosphorica (Vell.) H.Rob.

Z

A 11,0

Z

$\mathrm{Z} \quad 1,6$

Z

$\mathrm{U}$

A $\quad 31,4$

Z

A 75,1

A 254,0

1,0

7,2

0,2

0,2

0,4

0,2

Arbustos/subarbustos

Baccharis dracunculifolia DC.

Leandra australis (Cham.) Cogn.

$\begin{array}{cccc}\text { A } & 119,3 & & 69,4 \\ \text { Z } & 40,5 & 0,4 & 5,0\end{array}$

2,0

\section{Ervas}

Achyrocline satureioides (Lam.) DC.

Ageratum conyzoides L.

Amaranthus spinosus L.*

Andropogon bicornis L.

Andropogon leucostachyus Kunth

Asclepias curassavica L.

Baccharis trimera (Less.) DC.

Bidens pilosa L.

Baccharis crispa Spreng.

Centella asiatica (L.) Urban.

Chaptalia nutans (L.) Pol.

Chloris gayana Kunth*

Clidemia sp.

$\begin{array}{ccccccc}\text { A } & 1,9 & & & & & \\ \text { A } & 0,3 & 17,6 & & 0,6 & 66,2 & \\ \text { U } & 1,6 & 0,6 & & 0,9 & 2,7 & \\ \text { A } & 129,4 & & 0,4 & 456,3 & & 2,2 \\ \text { A } & 0,3 & & & & & 0,2 \\ \text { A } & & & & & & 0,2 \\ \text { A } & 0,3 & & & 2,2 & & \\ \text { Z } & & & & 6,0 & & \\ \text { A } & & 0,6 & & & 1,6 & \\ \text { U } & & 28,8 & 19,8 & & 22,4 & 9,6 \\ \text { A } & 3,5 & & & & & \\ \text { A } & 1,6 & 55,4 & 1,0 & & 23,0 & 0,4 \\ \text { Z } & & & 0,4 & & & 0,6 \\ \text { Z } & & & 0,8 & & & \\ \text { A } & & 25,0 & 0,4 & & 6,4 & 0,8 \\ \text { A } & 3,1 & & & & & \end{array}$

Coccocypselum sp

Commelina erecta $\mathrm{L}$.

Conyza bonariensis (L.) Cronq.

A $\quad 3,1$ 
Tabela 2 (continuação)

\begin{tabular}{|c|c|c|c|c|c|c|c|}
\hline \multirow{3}{*}{ Espécies/Species } & \multirow{3}{*}{ SD } & \multicolumn{3}{|c|}{ P1 } & \multicolumn{3}{|c|}{$\mathrm{P} 2$} \\
\hline & & \multicolumn{3}{|c|}{$\mathrm{DA}$} & \multicolumn{3}{|c|}{$\mathrm{DA}$} \\
\hline & & $\mathrm{CS}$ & BS & $\mathrm{RN}$ & $\mathrm{CS}$ & BS & $\mathrm{RN}$ \\
\hline Cyrtocymura scorpioides (Lam.) H. Rob. & A & & & 2,0 & & & 4,8 \\
\hline Cuphea carthagenensis (Jacq.) J.Macbr. & A & & 229,1 & 2,0 & & 152,0 & 2,0 \\
\hline Cyperus brevifoluus (Rottb.) Hassk. & A & & & & 8,2 & & \\
\hline Cyperus distans L.f. & A & & & & 9,7 & & \\
\hline Cyperus esculentus L.* & A & 5,0 & & & 252,2 & & \\
\hline Cyperus ferax Rich. & $\mathrm{A}$ & & & 0,2 & & & 0,2 \\
\hline Cyperus rotundus $\mathrm{L}$. & $\mathrm{A}$ & 44,9 & & & 3,1 & & \\
\hline Desmodium adscendens (SW.) DC.* & $\mathrm{Z}$ & 12,2 & & & 26,7 & & \\
\hline Desmodium barbatum (L.) Benth & $\mathrm{Z}$ & 1,6 & & 9,4 & 0,9 & & 15,8 \\
\hline Desmodium uncinatum (Jacq.) DC. & $\mathrm{Z}$ & & & 0,8 & & 0,6 & \\
\hline Borreria palustris (Cham. \& Schultdl.) Bacigalupo \& E.L.Cabral & A & & 66,6 & 8,6 & & 60,3 & 3,8 \\
\hline Elephantopus mollis Kunth & A & 7,2 & 1,0 & 0,6 & 0,6 & & \\
\hline Coniza bonariensis (L.) Cronquist & A & & & & & 0,6 & \\
\hline Chromolaena laevigata (Lam.) R.M.King \& H.Rob.. & $\mathrm{A}$ & 45,2 & & & 9,7 & & 0,4 \\
\hline Chromolaena squalida (DC.) R.M. King \& H.Rob. & A & 8,8 & & & & & \\
\hline Fimbristylis autumnalis (L.) Roem. \& Schult & A & & 41,6 & 3,2 & & 11,8 & 5,0 \\
\hline Fimbristylis dichotoma (L.) Vahl & A & & 868,8 & 1,2 & 11,0 & 730,2 & 0,2 \\
\hline Pterocaulon virgatum (L.) DC. & A & 5,3 & 0,6 & & 0,3 & 3,2 & 0,4 \\
\hline Pombalia communis (A St. -Hil.) Paula-Souza & $\mathrm{Z}$ & 33,0 & & & 4,1 & & \\
\hline Jaegeria hirta (Lag.) Less. & A & 3,8 & & & 0,3 & 7,5 & \\
\hline Lantana camara $\mathrm{L}$. & $\mathrm{Z}$ & & & & 0,3 & & \\
\hline Melilotus indicus (L.) All. & $\mathrm{Z}$ & 12,2 & & & 5,0 & & \\
\hline Melinis minutiflora P. Beauv.* & A & & & & 72,2 & & \\
\hline Megathyrsus maximus (Jacq.) B.K.Simon \& S.W.L.Jacobs & A & & & & 8,8 & & \\
\hline Panicum repens $\mathrm{L} . *$ & A & 2,8 & & & 176,5 & & \\
\hline Panicum sp. & A & 2,5 & & & 77,9 & 1,1 & \\
\hline Paspalum conspersum Schrad. & A & 28,6 & & & 513,4 & & 1,4 \\
\hline Paspalum sp. & A & 63,4 & & & 61,9 & & \\
\hline Phyllanthus tenellus Roxb. & A & & 11,7 & & & 1,1 & \\
\hline Sauvagesia erecta $\mathrm{L}$. & A & & & & & & 3,6 \\
\hline Scleria gaertneri Raddi & A & 3,8 & & 8,6 & 56,5 & 0,6 & 1,6 \\
\hline Scoparia dulcis L. & A & & 2,1 & & & 16,0 & 1,0 \\
\hline Setaria parviflora (Poir.) Kerguélen & $\mathrm{A}$ & & & & & & 0,2 \\
\hline Sida rhombifolia $\mathrm{L}$. & $\mathrm{Z}$ & 1,6 & 83,7 & 7,2 & 160,5 & 303,0 & 5,6 \\
\hline Sida sp. & $\mathrm{Z}$ & & & 1,2 & 53,4 & & \\
\hline Solanum americanum Mill. & $\mathrm{Z}$ & 4,4 & & & & & \\
\hline Stachytarpheta cayennensis (Rich.) Vahl & A & & 8,0 & & & 33,6 & 0,4 \\
\hline Urochloa decumbens (Stapf) R.D.Webster* & A & 1594,0 & 18,6 & 2,0 & 76,9 & 66,2 & 1,0 \\
\hline Urochloa mutica (Forssk.) T.Q. Nguyen* & A & & 24,5 & & 332,2 & 11,8 & \\
\hline \multicolumn{8}{|l|}{ Indeterminadas/Undetermined } \\
\hline Baccharis sp. & $\mathrm{A}$ & 105,5 & & & 25,4 & & \\
\hline Vernonia sp. & A & 30,5 & & & & & \\
\hline
\end{tabular}


Tabela 3. Número total de indivíduos, densidade absoluta e número de espécies encontradas na chuva de sementes, banco de sementes e estrato regenerante, na área de citricultura e na pastagem abandonadas no Parque Estadual do Jurupará, Ibiúna, SP. C: citricultura, P1: 0-20 m de distância do remanescente florestal, P2: 80-100 m de distância do remanescente florestal. DA: densidade absoluta (número de sementes $\mathrm{m}^{-2}$ ), $\mathrm{N}$ : número de indivíduos, Nsp: número de espécies.

Table 3. Total number of individuals, absolute density and number of species found in seed rain, seed bank and saplings in abandoned citrus crop and pasture of the Jurupará State Park, Ibiúna, SP. C: citrus crop, P1: 0-20 m distance of the forest remnant, P2: 80-100 $\mathrm{m}$ distance of the forest remnant. DA: absolute density (number of seeds $\mathrm{m}^{-2}$ ), $\mathrm{N}$ : number of individuals, Nsp: number of species.

\begin{tabular}{|c|c|c|c|c|c|c|}
\hline & \multicolumn{3}{|c|}{ Espécies nativas/exóticas } & \multicolumn{3}{|c|}{ Espécies Nativas } \\
\hline & $\mathrm{N}$ & $\mathrm{DA}$ & Nsp & $\mathrm{N}$ & $\mathrm{DA}$ & Nsp \\
\hline \multicolumn{7}{|c|}{ Chuva de sementes } \\
\hline $\mathrm{C}$ & 106.713 & 33.510 & 33 & 106.701 & 33.506 & 24 \\
\hline $\mathrm{P} 1$ & 8.570 & 2.691 & 37 & 3.415 & 1.072 & 30 \\
\hline $\mathrm{P} 2$ & 9.750 & 3.062 & 36 & 6725 & 2.111 & 29 \\
\hline Total & 125.033 & 13.088 & 53 & 116.841 & 12230 & 44 \\
\hline \multicolumn{7}{|c|}{ Banco de sementes } \\
\hline $\mathrm{C}$ & 3.133 & 1.671 & 28 & 1852 & 988 & 23 \\
\hline $\mathrm{P} 1$ & 2.793 & 1.490 & 20 & 2607 & 1390 & 16 \\
\hline $\mathrm{P} 2$ & 2.853 & 1.522 & 23 & 2659 & 1418 & 19 \\
\hline Total & 8.779 & 1.561 & 33 & 7118 & 1265 & 27 \\
\hline \multicolumn{7}{|c|}{ Estrato regenerante } \\
\hline $\mathrm{C}$ & 117 & 23 & 18 & 117 & 23 & 18 \\
\hline $\mathrm{P} 1$ & 356 & 71 & 24 & 341 & 68 & 22 \\
\hline P2 & 320 & 64 & 27 & 311 & 62 & 24 \\
\hline Total & 793 & 53 & 43 & 769 & 51 & 39 \\
\hline
\end{tabular}

fragmentadas ou cercadas de vegetação não natural (Garwood 1989, Dupuy \& Chazdon 1998, GromboneGuaratini \& Rodrigues 2002, Grombone-Guaratini et al. 2004). Alguns fatores, como a rápida germinação e maturação destas plantas (Hopkins et al., 1990, Christoffoleti \& Caetano 1998), mecanismos eficientes de dispersão e dormência colaboram para tal padrão (Vázquez-Yanes \& Orozco-Segovia 1987, Garwood 1989). Os resultados obtidos aqui, corroboram com a constatação de Baider et al. (2001), de que os bancos de sementes encontrados nos solos tropicais são, na sua maioria, constituídos por ervas, arbustos e árvores pioneiras. A baixa riqueza e densidade das espécies arbustivas e arbóreas encontradas no banco de sementes são recorrentes (Grombone-Guaratini \& Rodrigues 2002, Bertoncini \& Rodrigues 2008), segundo Janzen \& Vázquez-Yanes (1991) esta composição não é suficiente para restabelecer a diversidade e dinâmica de uma floresta.

A preponderância das espécies anemocóricas no banco de sementes em ambas as coberturas decorre de que as sementes anemocóricas frequentemente são pequenas e de baixa biomassa (Ressel et al. 2004). Harper (1977) e Baider et al. (2001) já relacionaram estas características com a formação de banco de sementes, entre as vantagens adaptativas, comumente conferidas a essa característica, estão: menor pressão de predação, menor vulnerabilidade ao ataque de fungos e maior facilidade de incorporação ao estoque do solo (Orozco-Segovia et al. 1993).

$\mathrm{O}$ estrato regenerante foi influenciado pela cobertura vegetal, as maiores densidades ocorreram na pastagem, em ambas as distâncias, em decorrência do predomínio de espécies herbáceas: Centella asiatica, Desmodium barbatrum, Scleria gaertneri, Sida rhombifolia, Fimbristylus autumnalis. Ao considerarmos apenas as espécies arbustivas arbóreas a densidade foi três vezes maior na citricultura, do que na pastagem. $\mathrm{O}$ estrato regenerante na pastagem não apresentou uma relação inversamente proporcional com a distância do remanescente florestal, indicando que a cobertura graminóide, praticamente uniforme 
em toda a pastagem, compete com as demais plantas e afeta diretamente o estabelecimento, sobrevivência e o crescimento de plântulas e indivíduos jovens nas pastagens, como observado por Hardwick et al. (1997) e Holl (2002). Essa competição atua de inúmeras formas, incluindo a disputa por luminosidade, água e nutrientes no solo, alelopatia química, ou por alterar a abundância e a atividade de herbívoros (Nepstad et al. 1991, Guariguata et al. 1995, Sun \& Dickinson 1996, Holl 2002). Holl (2002) destacou que a baixa luminosidade sob as gramíneas é a principal causa, já Uhl (1987) e Nepstad et al. (1991) sugeriram que altas temperaturas e baixa disponibilidade de umidade nas pastagens, principalmente no período mais seco, podem resultar em stress hídrico para as espécies lenhosas e limitar o estabelecimento e sobrevivência das plântulas. Diante dos inúmeros fatores, o grande desafio atual é separar os efeitos de cada um.

A síndrome de dispersão mais frequente entre os indivíduos regenerantes foi a zoocórica na área de citricultura e a anemocórica/autocórica na pastagem. A alta densidade e riqueza proporcional das espécies zoocóricas na área de citricultura, indica que o Citrus latifolia atuou como poleiro para a fauna, favorecendo a dispersão de sementes e o recrutamento de espécies zoocóricas, ao criar um micro-habitat favorável à regeneração destas espécies; Holl et al. (2000) em um estudo na Costa Rica e Günter et al. (2007) no sul do Equador, também obtiveram resultados semelhantes.

$\mathrm{Na}$ área com citricultura, dentre todas as plantas observadas no estrato em regeneração, apenas duas espécies: Commelina erecta e Aristolochia sp. foram registradas no banco de sementes. Essa situação provavelmente decorre da condição de sombreamento da área, que não é favorável à germinação de espécies heliófitas, que corresponderam a 99\% das espécies encontradas no banco de sementes. Estes resultados indicam que o dossel formado pela cultura de citrus, tem uma função importante na evolução da comunidade, reduzindo ou impedindo a germinação de parte das espécies intolerantes à sombra que chegam ao local, a partir da alteração das condições ambientais no seu subosque, exercendo, portanto o papel de filtro (Carnevale \& Montagnini 2002, Vieira \& Gandolfi 2006, Gandolfi et al. 2007b). Na pastagem, 100\% das espécies verificadas no banco de sementes foram encontradas na forma de plântulas, jovens ou adultos, provavelmente em virtude da condição de pleno sol desta área, Holl (1999) obteve resultado similar em um estudo na Costa Rica.
$\mathrm{Na}$ área de citricultura, além das diferenças quantitativas com a pastagem considerando o indicador chuva de sementes (31 vezes maior na citricultura), é importante destacar que tanto a chuva de sementes, quanto os indivíduos regenerantes apresentaram maior riqueza de espécies arbustivas arbóreas, indicando que a cobertura vegetal atuou como filtro e foi decisiva no processo de regeneração natural na área de citricultura.

A restauração florestal por meio da condução da regeneração natural em áreas degradadas é sem dúvida interessante, pois gera acréscimos evidentes para a biodiversidade local (Lamb 1998) e concilia a produção florestal com a restauração da biodiversidade (Carneiro \& Rodrigues 2007), devendo ser aproveitada nas diversas situações em que é viável do ponto de vista econômico e ambiental (Viani et al. 2010).

$\mathrm{O}$ fato de que após nove anos de abandono, ainda se mantém na pastagem, em ambas as distâncias, o predomínio de espécies herbáceas e baixa densidade de espécies arbustivas e arbóreas na regeneração natural também foi observado por Florentine \& Westbrooke (2004) que relacionaram às consequências das atividades de manutenção das pastagens, mesmo após anos de abandono, como o esgotamento dos nutrientes do solo, compactação o solo, favorecimento da aridez, perda de micorrizas, estabelecimento de espécies de gramíneas de crescimento rápido, prevalecendo condições adversas à regeneração natural por anos.

Liebsch et al. (2008) em um estudo realizado na Floresta Atlântica, analisaram os resultados de 18 levantamentos fitossociológicos realizados em áreas que sofreram corte, queima e subsequente utilização para atividades agropastoris e estimaram que em média são necessários 167 anos para que tais áreas retornem à condição de uma floresta madura e aproximadamente 2.000 anos para recuperar a taxa de $40 \%$ de endemismo da Floresta Atlântica. Duncan \& Chapman (1999) observaram, em um estudo no Kibale, que áreas degradadas por atividades agropastoris abandonadas evoluem lentamente, persistindo a pastagem mesmo depois de 100 anos de abandono. O recrutamento de plantas em áreas degradadas pode incluir poucas árvores e provavelmente áreas limitadas sob árvores médias e grandes. No entanto, áreas ao redor destas árvores podem ser dominadas por uma alta densidade de gramíneas, que podem impedir o estabelecimento das poucas sementes que chegam à área. Além disso, durante os períodos secos, estas pastagens frequentemente queimam, eliminando 
muitas árvores colonizadoras e perpetuando a dominância das gramíneas.

O processo de recuperação das pastagens abandonadas poder ser intensificado a partir da atração da avifauna. Reid \& Holl (2013) verificaram que o estabelecimento de espécies arbóreas, pode facilitar a recuperação, por aumentar a dispersão e sombrear as gramíneas na pastagem, no entanto, estruturas que atuam apenas como poleiros de pássaros não é uma estratégia efetiva, pois elas aumentam a chuva de sementes, mas não ajudam a ultrapassar os outros obstáculos, especialmente a competição com as gramíneas típicas da pastagem.

Além disso, há vários filtros desde a deposição dos diásporos até a fase de plântula, Holl \& Lulow (1997) verificaram que aproximadamente $2 / 3$ dos diásporos são removidos do local em que foram depositados em 30 dias, a partir do transporte vertical: atividades de vertebrados e a percolação da água da chuva. Desta forma, apenas 1/3 dos diásporos provenientes da chuva de sementes permanecem no solo, estes ainda podem sofrer com as condições microclimáticas adversas levando-as ao dessecamento (Uhl et al. 1981) ou a ação de patógenos (Augspurger 1984) antes de germinarem ou serem incorporados ao banco de sementes.

O restabelecimento da sucessão ecológica em áreas com um elevado grau de degradação, como a pastagem em questão, exige interferência. Ações de manejo que incluam o controle das espécies exóticas e o plantio de espécies nativas podem aumentar a cobertura arbustivo-arbórea e desencadear o processo de sucessão ecológica. O estabelecimento de uma cobertura arbustivo-arbórea, com estrutura equivalente a área de cultivo de citrus, facilitará o estabelecimento de propágulos originários do remanescente florestal próximo, contribuindo para o avanço da sucessão.

\section{Conclusões}

O tipo de cobertura vegetal foi determinante no processo de regeneração natural e atuou como catalisador da sucessão secundária, já que foram encontradas diferenças significativas na chuva de sementes entre a área de citricultura e a pastagem.

$\mathrm{Na}$ pastagem, a distância do remanescente florestal não foi significativa para a chuva de sementes, banco de sementes e estrato regenerante. Constatamos, nesta área, em ambas as distâncias do remanescente florestal baixa densidade de indivíduos arbustivoarbóreos para todos os indicadores.
O banco de sementes, não apresentou diferenças significativas entre as coberturas vegetais e distância do remanescente florestal; constatamos em ambas as situações o predomínio de espécies herbáceas e anemocóricas, próprias dos bancos de sementes de áreas alteradas.

As recomendações de manejo para as áreas são distintas, na área de citricultura é indicado apenas o monitoramento da regeneração natural, já na pastagem será necessário o plantio de mudas, com a finalidade do estabelecimento de uma cobertura arbustiva-arbórea com estrutura semelhante à da área de citricultura.

\section{Agradecimentos}

À FAPESP - Fundação de Amparo à Pesquisa do Estado de São Paulo, pela bolsa de estudos concedida (processo 00/04175-0). Ao Prof. Dr. Antônio Carlos Ricardo Braga Junior - UFBA pelo auxílio nas análises estatísticas. A toda equipe do Parque Estadual do Jurupará.

\section{Literatura citada}

Aide, T.M., Zimmerman, J.K., Pascarella, J.B., Rivera, L. \& Marcano-Vega, H. 2000. Forest regeneration in a chronosequence of tropical abandoned pastures: Implications for restoration ecology. Restoration Ecology 8: 328-338.

APG IV. 2016. An update of the Angiosperm Phylogeny Group classification for the orders and families of flowering plants: APG IV. Botanical Journal of the Linnean Society 181: 1-20.

Aubréville, A. 1963. Classification des formes biologiques des plantes vasculaires in milieu tropicale. Adansonia 3: 221-226.

Augspurger, C.K. 1984. Pathogen mortality of tropical tree seedlings: Experimental studies of the effects of dispersal distance, seedling density, and light conditions. Oecologia 61: 211-217.

Baider, C., Tabarelli, M. \& Mantovani, W. 2001. The soil seed bank during Atlantic Forest regeneration in southeast Brazil. Revista Brasileira de Biologia 61: 35-44.

Bertoncini, A.P. \& Rodrigues, R.R. 2008. Forest restoration in an indigenous land considering a forest remnant influence (Avaí, São Paulo State, Brazil). Forest Ecology and Management 255: 513-321.

Bossuyt, B., Honnay O. \& Hermy, M. 2005. Evidence for community assembly constraints during succession in dune slack plant communities. Plant Ecology 178: 201-209. 
Camus, J.M., Parrota, J., Brockerhoff, M.A., Jactel, H., Kremer, A., Lamb, D., O'Hara, K. \& Walters, B. 2006. Planted forests and biodiversity. Journal of Forestry 104: 65-77.

Carneiro, P.H.M. \& Rodrigues, R. R. 2007. Management of monospecific commercial reforestations for the forest restoration of native species with high diversity. In: R.R. Rodrigues, S.V. Martins \& S. Gandolfi (eds.). High Diversity Forest Restoration in Degraded Areas: Methods and Projects in Brazil. Nova Science Publishers, New York, pp. 129-144.

Carnevale, N.J. \& Montagnini, F. 2002. Facilitating regeneration of secondary forests with the use of mixed and pure plantations of indigenous tree species. Forest Ecology and Management 163: 217-227.

Christoffoleti, P.J. \& Caetano, R.S.X. 1998. Soil seed banks. Scientia Agricola 55: 74-78.

Departamento de Águas e Energia Elétrica - DAEE. 2017. Banco de dados hidrológicos. Disponível em http://www.hidrologia.daee.sp.gov.br/ (acesso em 12-II-2017).

Dalgaard, P. 2002. Introductory statistics with R. SpringerVerlag, New York.

Dosch, J.J., Peterson, C.J. \& Haines, B.L. 2007. Seed rain during initial colonization of abandoned pastures in the premontane wet forest zone of southern Costa Rica. Journal of Tropical Ecology 23: 151-159.

Duncan, R.S. \& Chapman, C.A. 1999. Seed dispersal and potential forest succession in abandoned agriculture in Tropical Africa. Ecological Applications 9: 998-1008.

Dupuy, J.M. \& Chazdon, R.L. 1998. Long-term effects of forest regrowth and selective logging on the seed bank of tropical forests in NE Costa Rica. Biotropica 30: 223-237.

Florentine, S.K. \& Westbrooke, M.E. 2004. Restoration on abandoned tropical pasturelands - do we know enough? Journal of Nature Conservation 12: 85-94.

Fundação Florestal do Estado de São Paulo (coord.). 2010. Plano de Manejo do Parque Estadual do Jurupará. Governo do Estado de - Secretaria do Meio Ambiente, São Paulo.

Gandolfi, S., Martins, S.V. \& Rodrigues, R.R. 2007a. Forest restoration: many views and objectives. In: R.R. Rodrigues \& S.V. Martins (eds.). High Diversity Forest Restoration in Degraded Areas. Nova Science Publishers, New York, pp. 3-26.

Gandolfi, S., Joly, C.A. \& Rodrigues, R.R. 2007 b. Permeability $\mathrm{x}$ impermeability: Canopy trees as biodiversity filters. Scientia Agricola 64: 433-438.

Garwood, N.C. 1989. Tropical soil seed banks: a review. In: M. Leck, V. Parker \& R. Simpson (eds.). Ecology of soil seed banks. Academic Press, San Diego, pp. 149-209.
Greene, D.F. \& Johnson, E.A. 1996. Wind dispersal of seeds from a forest into a clearing. Ecology 77:595-609.

Grombone-Guaratini, M.T. 1999. Dinâmica de uma floresta Estacional Semidecidual: o banco, a chuva de sementes e o estrato de regeneração. Tese de Doutorado, Universidade Estadual de Campinas, Campinas.

Grombone-Guaratini, M.T. \& Rodrigues, R.R. 2002. Seed bank and seed rain in a seasonal semi-deciduous forest in south-eastern Brazil. Journal of Tropical Ecology 18: 759-774.

Grombone-Guaratini, M.T., Filho, H.F.L. \& Kageyama, P.Y. 2004. The seed bank of a gallery forest in Southeastern Brazil. Brazilian Archives of Biology and Technology 47: 793-797.

Guariguata, M.R., Rheingans, R., Montagnini, F. 1995. Early woody invasion under tree plantations in Costa Rica: implications for forest restoration. Restoration Ecology 3: 252-260.

Günter, S., Weber, M., Erreis, R., Aguirre, N. 2007. Influence of distance to forest edges on natural regeneration of abandoned pastures: a case study in the tropical mountain rain forest of Southern Ecuador. European Journal of Forest Research 126: 67-75.

Harper, J.L. 1977. Population biology of plants. Academic Press, London.

Hardwick, K., Healey, J., Elliott, S., Garwood, N., \& Anusarnsunthorn, V. 1997. Understanding and assisting natural regeneration processes in degraded seasonal evergreen forests in northern Thailand. Forest Ecology and Management 99: 203-214.

Heerdt, G.N.J., Verweij, G.L., Bakker, R.M. \& Bakker, J.P. 1996. An improved method for seed bank analysis: seedling emergence after removing the soil by sieving. Functional Ecology 10: 144-151.

Holl, K.D. 1999. Factors limiting tropical rain forest regeneration in abandoned pasture: seed rain, seed germination, microclimate and soil. Biotropica 31: $229-242$.

Holl, K.D. 2002. Effect of shrubs on tree seedling establishment in an abandoned tropical pasture. Journal of Ecology 90: 179-187.

Holl, K.D. \& Lulow, M.E. 1997. Effects of species, habitat, and distance from edge on post-dispersal seed predation in a tropical rainforest. Biotropica 29: 459-468.

Holl, K.D., Loik, M.E., Lin, E.H.V. \& Samuels, I.A. 2000. Tropical montane forest restoration Costa Rica: Overcoming barriers to dispersal and establishment. Restoration Ecology 8: 339-349.

Hopkins, M.S., Tracey, J.G. \& Graham, A.W. 1990. The size and composition of soil seed banks in remnant patches of three structural rainforest types in North Queensland, Australia. Melbourne. Australian Journal of Ecology 15: 43-50. 
Howe, H.F. \& Smallwood, J. 1982. Ecology of seed dispersal. Annual Review of Ecology Systematics 13: 201-228.

Hubbell, S.P. 2001. The unified neutral theory of biodiversity and biogeography. Princeton University Pres, New Jersey.

Ingle, N.R. 2003. Seed dispersal by wind, birds, and bats between Philippine montane rainforest and succesional vegetation. Oecologia 134: 251-261.

Janzen, D.H. \& Vásquez-Yanes, C. 1991. Aspects of tropical seed ecology of relevance to management of tropical forested wildlands. In: M. Hadley (ed.). Rain Forest Regeneration and Management. Unesco, Paris, pp. 28-33.

Kim, A.C. 1996. Lianas da mata atlântica do Estado de São Paulo. Dissertação de Mestrado, Universidade Estadual de Campinas, Campinas.

Köppen, W.P. 1948. Climatologia. Fondo de Cultura Economica, Mexico.

Lamb, D. 1998. Large-scale ecological restoration of degraded tropical forest lands: The potential role of timber plantations. Restoration Ecology 6: 271-279.

Liebsch, D., Marques, M.M.C. \& Goldenberg, R. 2008. How long does the Atlantic Rain Forest take to recover after a disturbance? Changes in species composition and ecological features during secondary succession. Biological Conservation 141: 1717-1725.

Lima, R.A.F. \& Aguirre, A.G. 2010. Vegetação e Flora. In: Fundação Florestal do Estado de São Paulo (coord.). Plano de Manejo do Parque Estadual do Jurupará. Governo do Estado - Secretaria do Meio Ambiente, São Paulo, pp.122-134.

Lista de Espécies da Flora do Brasil. 2016. Jardim Botânico do Rio de Janeiro. Disponível em http:// floradobrasil.jbrj.gov.br/ (acesso em 16-II-2016).

Lopes, S.F., Oliveira, A.P., Neves, S.B. \& Schiavini, I. 2010. Dispersão de sementes uruvalheira (Platypodium elegans Vog.) (Fabaceae) em um Cerradão, UberlândiaMG. Revista Árvore 34: 807-813.

Nepstad, D.C., Uhl, C. \& Serrão, E.A.S. 1991. Recuperation of a degraded Amazonian landscape: forest recovery and agricultural restoration. Ambio 20: 248-255.

Obiri, J.A. \& Lawes, M.J. 2004. Chance versus determinism in canopy gap regeneration in coastal scarp forest in South Africa. Journal of Vegetation Science 15: 539-547.

Orozco-Segovia, A., Sanchez-Coronado, M.E. \& Vázquez-Yanes, C. 1993. Light environment and phytochrome - controlled germination in Piper auritum. Functional Ecology 7: 585-590.

R Core Team. 2016. R: The R Project for statistical computing. Disponível em https://www.r-project.org (acesso em 04-IV-2016).
Reid, J.L. \& Holl, K.D. 2013. Arrival $\neq$ Survival. Restoration Ecology 21: 153-155.

Ressel, K., Guilherme, R.A.G., Schiavini, I. \& Oliveira, P.E. 2004. Ecologia morfofuncional de plântulas de espécies arbóreas da Estação Ecológica do Panga, Uberlândia, Minas Gerais. Revista Brasileira de Botânica 27: 311-323.

Rodrigues, R.R., Brancalion, P.H.S. \& Isernhagen, I. 2009. Pacto para a restauração da Mata Atlântica: referencial dos conceitos e ações de restauração florestal. Instituto BioAtlântica, São Paulo.

Silva Júnior, M.C., Scarano, F.R. \& Cardel, F.S. 1995. Regeneration of an Atlantic Forest in the understory of an Eucalyptus grandis stand in southern Brazil. Journal of Tropical Ecology 11:148-152.

Slocum, M.G. \& Horvitz, C.C. 2000. Seed arrival under different genera of trees in a neotropical pasture. Plant Ecology 149: 51-62.

Sun, D. \& Dickinson, G.R. 1996. The competition effect of Brachiaria decumbens on the early growth of directseeded trees of Alphitonia petriei in tropical north Australia. Biotropica 28: 272-276.

Tabarelli, M., Villani, J.P. \& Mantovani, W. 1993. A recuperação da floresta atlântica sob plantios de Eucalyptus no núcleo Santa Virgínia, SP. Revista do Instituto Florestal 5: 187-201.

Uhl, C. 1987. Factors controlling successsion following slash-and-burn agriculture. Journal of Ecology 75: 377-407.

Uhl, C., Clark, K., Clark, H. \& Murphy, P. 1981. Early plant succession after cutting and burning in the upper Rio Negro region of the Amazonian Basin. Journal of Ecology 69: 631-649.

Uhl, C., Buschbacher, R. \& Serrão, E.A.S. 1988. Abandoned pastures in eastern Amazonia. I. patterns of plant succession. Journal of Ecology 76: 663-681.

Uhl, C., Nepstad, D., Silva, J.M.C. \& Vieira, I. 1991. Restauração da floresta em pastagens degradadas. Ciência Hoje 13: 22-31.

Van der Pijl, L. 1982. Principles of dispersal in higher plants. 2 ed. Springer-Verlag, Berlim.

Vázquez-Yanes, C. \& Orozco-Segovia, A. 1987. Fisiologia ecologica de semillas en la Estacion de Biologia Tropical "Los Tuxtlas", Veracruz, Mexico. Revista de Biologia Tropical 35:85-89.

Viani, R.A.G., Durigan, G. \& Melo, A.C.G. 2010. A regeneração natural sob plantações florestais: desertos verdes ou redutos de biodiversidade? Ciência Florestal 20: 533-552.

Vieira, D.C.M. \& Gandolfi, S. 2006. Chuva de sementes e regeneração natural sob três espécies arbóreas em uma floresta em processo de restauração. Revista Brasileira de Botânica 29: 541-554. 